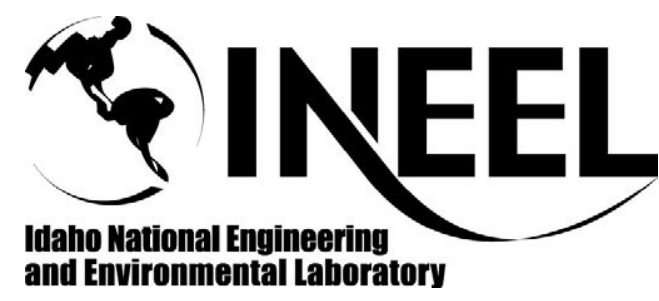

INEEL/CON-01-00984

PREPRINT

Microtopography For Ductile Fracture Process Characterization

Part 2: Application For CTOA Analysis

W. R. Lloyd

F. A. McClintock

April 24-26, 2001

Workshop On The Fundamentals And Application Of The Crack Tip Opening Angle (CTOA)

This is a preprint of a paper intended for publication in a journal or proceedings. Since changes may be made before publication, this preprint should not be cited or reproduced without permission of the author.

This document was prepared as an account of work sponsored by an agency of the United States Government. Neither the United States Government nor any agency thereof, or any of their employees, makes any warranty, expressed or implied, or assumes any legal liability or responsibility for any third party's use, or the results of such use, of any information, apparatus, product or process disclosed in this report, or represents that its use by such third party would not infringe privately owned rights. The views expressed in this paper are not necessarily those of the U.S. Government or the sponsoring agency. 


\title{
Microtopography for Ductile Fracture Process Characterization Part 2: Application for CTOA Analysis
}

\author{
W. R. Lloyd \\ Advisory Engineer \\ Idaho National Engineering and Environmental Laboratory \\ P.O. Box 1625, Idaho Falls, ID 83415-2218 \\ 208-526-0808, FAX 208-526-5327, <qrl@inel.gov> \\ F. A. McClintock \\ Professor Emeritus \\ Massachusetts Institute of Technology \\ 77 Massachusetts Ave., Rm 1-304, Cambridge, MA 02139 \\ 617-253-2219, FAX 617-258-8742, <fammam@aol.com>
}

\begin{abstract}
The crack tip opening angle (CTOA) is seeing increased use to characterize fracture in so-called "low constraint" geometries, such as thin sheet aerospace structures and thin-walled pipes. With this increase in application comes a need to more fully understand and measure actual CTOA behavior. CTOA is a measure of the material response during ductile fracture, a "crack tip response function." In some range of crack extension following growth initiation, a constant value of CTOA is often assumed. However, many questions concerning the use of CTOA as a material response-characterizing parameter remain. For example, when is CTOA truly constant? What three-dimensional effects may be involved (even in thin sheet material)? What are the effects of crack tunneling on general CTOA behavior? How do laboratory specimen measurements of CTOA compare to actual structural behavior?

Measurements of CTOA on the outer surface of test specimens reveal little about threedimensional effects in the specimen interior, and the actual measurements themselves are frequently difficult. The Idaho National Engineering and Environmental Laboratory (INEEL) use their microtopography system to collect data from the actual fracture surfaces following a test. Analyses of these data provide full three-dimensional CTOA distributions, at any amount of crack extension. The analysis is accomplished using only a single specimen and is performed entirely after the completion of a test. The resultant CTOA distributions allow development of full and effective understanding of CTOA behaviors. This paper presents underlying principles, various sources of measurement error and their corrections, and experimental and analytical verification of CTOA analysis with the microtopography method.
\end{abstract}

Keywords

Microtopography, ductile fracture, three-dimensional effects, CTOD, CTOA

\section{Introduction}

In the case of many structural components, the geometry of the structure near cracks causes reduction of the level of crack tip constraint, i.e. reduction of the normalized level of hydrostatic stress. This, in turn, invalidates a plane strain fracture mechanics analysis based on the J-integral or crack tip opening displacement (CTOD). For these low constraint conditions, the crack tip 
opening angle (CTOA) is seeing increased use as a parameter that characterizes the material response in the ductile fracture process. Investigations over the past ten years by different researchers, e.g. Dawicke et al. [1], Sutton et al. [2], and Lloyd and Piascik [3] show that the CTOA is a credible parameter that adequately characterizes the ductile fracture material response following crack initiation.

\section{Experimental Measurement Methods}

In the search for a simple characterizing parameter, a steady-state value of CTOA that is a characteristic of the material and geometry being analyzed is usually sought. Since the geometries of interest are often thin compared to the growing crack length, a common approach for measuring CTOA during an experiment involves making images of the lateral surfaces ${ }^{\mathrm{a}}$ of a test specimen during crack growth. Photographic images are collected at increasing lengths of the growing crack, and CTOA values are determined for each length. A steady-state average value is normally determined from these data. Other measurement methods, including the $\delta_{5} \mathrm{R}$ curve method and extrapolations from crack mouth opening displacement (CMOD) measurements are also employed. However, there are some potential sources of problems in all of these methods.

All of the aforementioned methods rely on measurements at the outer surface of a test specimen to infer some through-thickness average value of CTOA. Investigations by Lloyd and Piascik [3] and Sutton et al. [2] clearly show that CTOA can have strong dependence on through-thickness position, even in thin sheet materials. Their work also show substantial transient variation in CTOA immediately following crack initiation until the crack has advanced - at the specimen surface - the order of one sheet thickness in 7075 aluminum alloy. In addition, crack tunneling may proceed in the early stages of growth such that the difference in crack length between the surface and mid-thickness is nearly as much as the thickness of the sheet test specimen. All of these observations suggest that CTOA measurements based solely on observations of the outer surface of test specimens may include significant errors, especially in the early stages of crack growth.

Around 1989, about the same time of investigation into CTOA as a crack growth parameter ${ }^{\mathrm{b}}$ by Sutton et al [2] and Guan et al. [5] (others had explored CTOA before this time), INEEL developed the microtopography system of measurement and analysis. Microtopography is a unique method for assessing the deformations associated with the ductile fracture process. Topographic-type data (surface height versus planar position) are collected from fracture surfaces of test specimens or actual failed structures. Subsequently, the analysis method developed at INEEL is applied, allowing all of the material deformation-related aspects of the ductile fracture process to be extracted from these data. A significant feature of the method is its application occurs entirely after the completion of the ductile fracture process. The initial development of this capability was directed to investigation of crack initiation tip opening displacement $\left(\mathrm{CTOD}_{\mathrm{i}}\right)$, and CTOD variations for part-through surface cracks. Because of the broad general capabilities of the microtopography methodology to interrogate ductile fracture, we expanded the analysis methods to extract CTOA.

a the "faces" of a thin specimen.

b more accurately, a "crack tip material response function," dependent on the material and the stress distribution in the vicinity of the crack tip. 
The three-dimensional, post-test measurement features of the methodology allow the CTOA to be extracted from any location along the crack border, and at any point in the fracture process, i.e. at any amount of crack extension $(\Delta \mathrm{a})$, leading to a full characterization of the crack tip material response. Correlations to CMOD can also be made, allowing CMOD to become a fourth variable in the multi-dimensional data set. Through this analysis, the entire picture of the ductile crack growth process is available. This is proving to be an important capability for finite element modeling/analysis (FEM, FEA) of ductile fracture, especially where two-dimensional models are considered. Some suitable through-thickness average crack length and CTOA must be devised for comparison, since James and Newman [6] have demonstrated the relative insensitivity of the CMOD or load-line specimen displacements - typical points of comparison for FEA - to crack tunneling. The microtopography methodology capability for detailed three-dimensional assessments of CTOA proves it a powerful research tool for ductile fracture process characterization.

\section{CTOA Fundamentals}

The CTOA parameter - its function, description, and investigation - is grounded in the kinematics of plastic deformation. From an engineering perspective, using CTOA as an applied crack tip response parameter requires some fairly simple and readily applicable definition. There are various definitions of CTOA, usually based on the method of experimental measurement. These are detailed in Sutton et al. [2], Lloyd and Piascik [3], Zerbst et al. [7], and others. The most widely accepted definition measures the opening displacement between the crack flanks at a distance of $1 \mathrm{~mm}$ behind the instantaneous crack tip location. The CTOA is then defined by:

$$
C T O A=2 * \operatorname{atan}\left(\frac{1}{2} \cdot \frac{d}{x}\right)
$$

In the normal definition, $d$ is measured $1 \mathrm{~mm}$ behind the apparent crack tip, and $x$ is set at $1 \mathrm{~mm}$. Zerbst and Schwalbe [8] have defined CTOA based on average crack extension ${ }^{\mathrm{c}}$ and the $\delta_{5}$ crack tip opening measurement. In this case, $d$ is the $\delta_{5}$ tip opening and $x$ is the instantaneous $\Delta \mathrm{a}$. Additional modifications associated with mixed mode loading adopt the opening displacement aligned normal to the instantaneous crack growth direction. This modification shows promise in providing a more consistent CTOA value for slanted crack growth when compared to the normal CTOA measured under pure mode I crack opening.

The problems with surface measurements associated with through-thickness variation was discussed above. The $\delta_{5}$-based definition has additional variabilities related to the varying "gage length," $x$, and antisymmetric rigid specimen rotations that occur in typical test specimens like the $\mathrm{C}(\mathrm{T})$ or $\mathrm{SE}(\mathrm{B})$ geometries. These rotation effects are discussed in detail later, in the section "Kinematic Considerations - Anti-symmetric Rotation and Plastic Flow."

\section{Conventional Data Collection and Analysis Methods}

Regardless of the CTOA definition applied, some type of crack opening displacement must be measured at various increments of crack extension. The optical microscopy technique applied to measurement on the lateral, or face, surface requires manual measurement of crack opening at the

\footnotetext{
${ }^{\mathrm{c}}$ as determined by standard measurement techniques, see the appendix in ASTM Standard E 1820 [10]
} 
selected distance behind the apparent instantaneous crack tip on the collected images. Sutton et al. [2] and others note that locating the crack tip accurately is sometimes difficult due to image defocus and/or illumination problems. For the same reasons, the crack flank edge profiles may be equally difficult to locate precisely. Very rough edges compound these difficulties. Sutton's digital image correlation approach [2] eliminates some of these difficulties by measuring surface displacements on the lateral face at positions immediately above and below the crack flank edges. The technique also reduces some of the human error and subjectivity in locating and measuring the flank edge position. The method still suffers from the same crack tip locating problems. Zerbst and Schwalbe's [8] $\delta_{5}$ approach makes opening displacement measurements with a special type of clip gage transducer, but only at a fixed location - above and below the original crack tip at the specimen lateral surface. The discussion in the Introduction section identified some of the difficulties in using these approaches. While the basic concept of CTOA is a simple one, the actual measurement of its value has various difficulties associated with it - some more obvious, others rather subtle.

The "conventional" methods of CTOA determination - optical microscopy, digital image correlation, and the $\delta_{5}$ crack tip displacement method generally provide similar values of steadystate CTOA. They all have the potential to misinterpret actual average CTOA in early crack growth. The surface measurement is not usually representative of interior behavior, and crack growth initiation at the surface can be delayed relative to initiation in the specimen interior. The $\delta_{5}$ method, by its measurement definition, may over-predict CTOA at larger crack extension in test specimens that have significant bend-type loading. The microtopography methodology offers benefits related to several aspects of CTOA assessment. The two most significant benefits from the experimental viewpoint are collection of all data after the conclusion of the test, and the ability to measure CTOA in the specimen interior.

\section{3-D Aspects of CTOA Assessment}

INEEL, National Aeronautics and Space Administration (NASA), and University of South Carolina (USC) used various measurement techniques to determine the CTOA in $2.5 \mathrm{~mm}$ thick, 7075 aluminum $\mathrm{M}(\mathrm{T})$ specimens $[2,3]$. These specimens simulated cracks growing in aircraft skin material. The combined studies showed that CTOA has significant three-dimensional positional dependency, and strong dependency on local crack extension, even in the thin sheet specimens. These specimens exhibited crack tunneling exceeding the plate thickness. It is obvious that inferring a through-thickness average CTOA from an outside surface measurement, especially in the early stages of crack growth, will result in poor estimates of "average" CTOA behavior in this case of severe tunneling. CTOA was much higher at the surface than in the specimen interior immediately after crack initiation. Elevated constraint at mid-thickness, where the crack plane was normal to the surface plane (flat fracture mode) would be expected to cause decreased CTOA. As the crack grew, the fracture mode transitioned to full slant fracture, where a decrease in constraint at mid-thickness would be expected. The slight rise in measured midthickness CTOA with increasing crack growth correlates to the expected change in constraint.

These studies of the same physical test specimens [2,3], performed independently by INEEL and University of South Carolina showed CTOA at small $\Delta$ a were different in the interior than on the surface. CTOA assessed by both approaches - microtopography and surface imaging converged toward similar values with increasing crack extension. However, these "steady state" values were still slightly different. The surface-measured CTOA approached $4.7 \mathrm{deg}$ from a much higher initial value, while the interior CTOA measured by microtopography approached 5.3 deg from an initial value less than $5 \mathrm{deg}$. This example demonstrates the variability in CTOA 
with through-thickness position and crack extension, and shows the potential complexity involved in making an accurate assessment of the ductile fracture process.

\section{CTOA Analysis By Microtopography}

Lloyd provides data collection and methodology fundamentals of microtopography in the companion article [9]. In that article, the author demonstrates that deformation associated with ductile fracture occurs predominantly within the fracture process zone surrounding the crack tip. As the crack extends, the material deformation that creates the new fracture surfaces remains in those surfaces. The fracture surfaces see little, if any, further plastic deformation since normal and shear tractions there are approximately zero and the stress parallel to those surfaces are relatively low. By this reasoning, the ductile fracture process history is "recorded" in the fracture surfaces created as the crack grows. Microtopography uses this concept to recreate the ductile fracture process and extract process parameters.

\section{Mathematical Treatment of the Analysis Approach}

Using any suitable method, two discretely defined surfaces, $\mathrm{U}^{\prime}(\mathrm{x}, \mathrm{y})$ and $\mathrm{L}^{\prime}(\mathrm{x}, \mathrm{y})$, corresponding to upper and lower fracture surfaces are obtained. Investigations and experiments at INEEL suggest that spatial increments in y (crack growth direction) of $0.1 \mathrm{~mm}$ are normally adequate for the CTOA analysis. Height resolution (crack opening direction) must be appropriate for adequate accuracy of crack surface height measurement. Lower nominal CTOA dictates finer resolution in height measurement. Height resolution of about $1 \mu \mathrm{m}$ has been more than adequate for all cases studied to date. The $\mathrm{x}$ and $\mathrm{y}$ coordinates of the two data sets must be appropriately registered such that common points of instantaneous material separation on the two surfaces correlate in $(\mathrm{x}, \mathrm{y})$ space.

A height difference surface is then defined asl:

$$
\mathrm{D}_{\mathrm{j}}(\mathrm{x}, \mathrm{y})=\left(\mathrm{U}_{0}(\mathrm{x}, \mathrm{y}) \bullet \mathrm{P}_{\mathrm{j}}\left(\mathrm{a}_{\mathrm{j}}, \mathrm{y}\right) / 2\right)-\left(\mathrm{L}_{0}(\mathrm{x}, \mathrm{y}) \bullet \mathrm{P}_{\mathrm{j}}\left(\mathrm{a}_{\mathrm{j}}, \mathrm{y}\right) / 2\right)+\left(\mathrm{z}_{\mathrm{j}-1}+\Delta \mathrm{z}_{\mathrm{j}}\right) .
$$

where:

$\mathrm{j} \quad$ is an index representing an increment of crack opening, $\Delta \mathrm{z}$, and corresponding crack extension, $\Delta \mathrm{a}$;

$\mathrm{L}_{0}(\mathrm{x}, \mathrm{y})=\mathrm{L}^{\prime}(\mathrm{x}, \mathrm{y})$, the initial lower surface;

$\mathrm{U}_{0}(\mathrm{x}, \mathrm{y})=\mathrm{U}^{\prime}(\mathrm{x}, \mathrm{y})-\mathrm{t}(\mathrm{x}, \mathrm{y})$, the initial upper surface tilted to match at the fatigue precrack region;

$\mathrm{t}(\mathrm{x}, \mathrm{y}) \quad$ is a planar surface tilt correction function; ${ }^{\mathrm{d}}$

$\mathrm{P}_{\mathrm{j}}\left(\mathrm{a}_{\mathrm{j}}, \mathrm{y}\right)$ is the global specimen rotation correction term (angular correction function).

\footnotetext{
${ }^{d} t(x, y)$ is defined such that the initial difference surface, $D_{0}(x, y)$ is nominally 0 in the region of the fatigue precrack.
} 
$\mathrm{P}_{\mathrm{j}}\left(\mathrm{a}_{\mathrm{j}}, \mathrm{y}\right)$ provides a planar rotation about the $\mathrm{x}$-axis, centered at the incremental specimen center of rotation, $R_{j} . P$ is a linear function of $a_{j}$ and $y$, and $P_{0}=0$. The initial state, $D_{0}(x, y)$, prior to crack opening and advance is thus defined, as in Figure 1.

Figure 1 shows representative data from a section profile taken normal to the (x,y) plane and parallel to the local crack growth direction. The index, $\mathrm{j}$, corresponds to a selected state in the fracture process. Figure 1 represents $D$ when $\mathrm{j}=0$ - the initial state with precrack region closed. $\mathrm{D}_{\mathrm{j}}$ values less than 0 (nominal) have no physical meaning. Lloyd discusses this region representing a "virtual overlap" of material ahead of the instantaneous crack tip [9]. This region, which changes and diminishes as the crack growth process is advanced (as shown in Figures 2 5 ), is representative of the crack opening volume created as crack growth continues beyond the instantaneous state being analyzed.

Referring to Figure 5, $\mathrm{D}_{\mathrm{f}}(\mathrm{x}, \mathrm{y})=\left(\mathrm{U}_{0} \bullet \mathrm{P}_{\mathrm{j}}\left(\mathrm{a}_{\mathrm{j}}, \mathrm{y}\right) / 2\right)-\left(\mathrm{L}_{0} \bullet \mathrm{P}_{\mathrm{f}}\left(\mathrm{a}_{\mathrm{f}}, \mathrm{y}\right) / 2\right)+\left(\mathrm{z}_{\mathrm{f}-1}+\Delta \mathrm{z}_{\mathrm{f}}\right)$, is used to define $P$ such that the difference, $D_{f}$, is nominally 0 in the post-test elastic fracture (cleavage or fatigue) region. $P_{j}$ is 0 for symmetric specimens, e.g. $\mathrm{M}(\mathrm{T})$, with nominally symmetric crack growth. $\mathrm{D}_{0}$ (Figure 1) and $\mathrm{D}_{\mathrm{f}}$ (Figure 5) represent the two reference states in the crack growth process, initial and final respectively.

For a given fracture process state, $\mathrm{j}$, the incremental crack length, $\mathrm{a}_{\mathrm{j}}(\mathrm{x})$, is located at the $y$ value where $D_{j}(x, y)=0$ (the incremental crack tip border on the $x-y$ plane). A sequence of $D_{j}$ profiles is shown in Figures 1 - 5, with parameters identified.

Figure 2 represents the process state where crack initiation is just about to occur. At this state, the initiation value of CTOD can be easily determined by assessing $\mathrm{D}$ at $\Delta \mathrm{a}=0$. Figure 3 shows the $\mathrm{D}$ distribution at the point where $1.0 \mathrm{~mm}$ of ductile tearing has occurred. This state would be the point of first valid CTOA measurement if the $1 \mathrm{~mm}$ gage length definition of CTOA was to be applied. Figure 4 shows a subsequent state where about $2 \mathrm{~mm}$ of ductile tearing has occurred. Figure 5 represents the test end point for this particular data, where the test was ended by liquid nitrogen quenching and subsequent loading to cause a cleavage fracture.

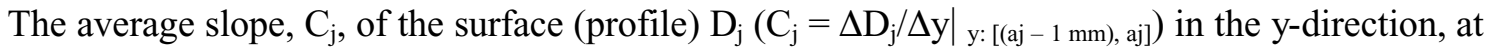
the $x$ location of choice (typically mid-thickness), in the range ${ }^{e} y:\left[\left(a_{j}-1 \mathrm{~mm}\right), a_{j}\right]$, where $y=\Delta a_{j}$ is the instantaneous crack tip position, relates to the incremental CTOA by

$$
\mathrm{CTOA}_{\mathrm{j}}=2 * \arctan \left(-\mathrm{C}_{\mathrm{j}} / 2\right) \text {. }
$$

Equation 3 is Equation 1 recast in terms used in Equation 2. A linear least squares fit of the discrete height data in the defined range of $\mathrm{y}$ defines the average slope, $\mathrm{Cj}$. This is shown graphically in Figures 3 and 4. Clearly, the data should be examined for outlying points (points not within the general trend of $\mathrm{D}$, that may perturb the CTOA results), or the $\mathrm{D}_{\mathrm{j}}$ function can be smoothed before analysis to eliminate measurement error and minor data point correlation errors. The resultant data, $C_{T O A}$ vs. $\Delta \mathrm{a}_{\mathrm{j}}$, can thus be developed, plotted, and analyzed. Such an analysis is demonstrated in Figure 6, based upon data from Figures 2 - 5, and CTOA values derived from process states in between those shown in the Figures.

\footnotetext{
${ }^{\mathrm{e}}$ this range of $1 \mathrm{~mm}$ behind the instantaneous crack tip is suggested in Reference 2 and in a draft ISO test standard, TC 164 SC-N.
} 
Note that early crack opening is associated with plastic tip blunting ("stretching," "stretch zone," "blunting zone," see Figure 2). CTOD is the defining parameter in that region, and CTOA has no practical significance before the onset of ductile tearing. Inferring CTOA from $\mathrm{C}_{\mathrm{j}}$ measurements in this region without regard for the deformation process will lead to faulty results, due to the blunting and nonlinear nature of the $\mathrm{D}$ function in this zone. CTOD is only calculated from $\mathrm{C}_{\mathrm{j}}$ determined from data collected within the tearing zone region (e.g. see Figure 3). The transition from blunting to stable tearing is normally identified by a change from rapidly increasing $\left|\mathrm{C}_{\mathrm{j}}\right|$ to a significantly lower, more slowly changing $\left|C_{j}\right|$.

The various curves in Figures 1 - 5 represent mathematical manipulation of the height data from the two mating surfaces according to Equation 2 to recreate the ductile fracture process. Very small increments of crack opening/advance allow the recreation to proceed in an essentially continuous manner. In an actual complete analysis, the process represented between Figure 1 and Figure 5 would include the order of 80 increments corresponding to $\triangle \mathrm{CTOD} \cong 20 \mu \mathrm{m}$. This, in turn, allows measurement of the crack front shape and local CTOA at very small crack extension increments when necessary.

Because the full, three-dimensional crack opening profiles are available at any point in the ductile fracture process, any definition of CTOA can be applied to extract the desired CTOA response parameter from the specimen data. The research presented in Reuter et al. [4] shows that microtopography has the added benefit of revealing meso-scale fracture process variability, e.g. crack tip zig-zagging, in the specimen interior. While such effects or behaviors are at a smaller size scale than the typical CTOA size regime of about $1 \mathrm{~mm}$, they do provide additional insight into the ductile fracture process. Microtopography provides a method to directly measure these process behaviors for study of ductile fracture at smaller size scales. However, the data from the two fracture surfaces must be manipulated as separate data sets to retain these features. When the height difference, $\mathrm{D}$, is calculated, these features are eliminated from the resultant combined data.

\section{Kinematic Considerations - Anti-symmetric Rotation and Plastic Flow}

Geometrically symmetric specimens, such as the $\mathrm{M}(\mathrm{T})$ plate specimen discussed above in " $3-D$ Aspects of CTOA Assessment," provide good baseline data to assess a measurement method, since there is no global anti-symmetric specimen rotation. The $\mathrm{M}(\mathrm{T})$ specimen was originally chosen because it accurately simulated the structural cracking of interest. However, it also allowed microtopography analysis to be applied to CTOA measurement without any antisymmetric specimen rotation corrections. Typical fracture toughness specimens [SE(B), C(T)] may experience large rotations during crack extension, especially with the higher-toughness materials where extensive stable tearing may be encountered.

Microtopography uses fracture surface data collected at the end of crack growth, so these antisymmetric rotations are embedded within the collected data. An approach based upon the rotation correction equations used in the CTOD fracture toughness test method - ASTM E 1820 [10] - is used in the microtopography analysis to achieve correct crack opening profiles at the various amounts of re-created crack extension. The "correct" amount of relative rotation between the upper and lower surfaces is known at two process states. At the "initial" state, the surfaces are known to match $^{\mathrm{f}}$ in the fatigue precrack region (Figure 1). At the "final" state, defined by post-test fatigue or post-test cleavage, the surfaces also are known to match (Figure 5). In most

\footnotetext{
${ }^{\mathrm{f}}$ when properly aligned, the height difference between the surfaces is near zero over the entire region.
} 
instances, some relative rotation between the upper and lower surface data sets are required in addition to uniform opening translation to create the height matching correlation at the two states.

The rotational difference between these two states is the total amount that occurs during the blunting and tearing process. The rotation correction applied at each crack opening/crack extension increment is calculated based on the instantaneous (at the current increment) center of rotation position, similar to that described in ASTM E 1820. The position of the rotation center is adjusted from the E 1820-calculated value, such that the total amount of accumulated rotation at the conclusion of the process re-creation correctly aligns the surfaces in the final state. The modification to the E 1820 location is dependent on several factors. Crack front curvature is an obvious consideration, as is nominal crack depth. The E 1820 equations are only applicable to a specified range of average crack length, while this method of analysis has no such limitation. Equation 4 is the basic equation used to manipulate the raw microtopography data to recreate the fracture process.

$$
Z_{i+1}=\left(Z_{i}-\frac{C T O D \text { incr }}{2}\right)+\operatorname{slope}\left(Y_{i}-R_{i+1}\right)
$$

where:

$$
\begin{aligned}
& Z \quad \text { are the height vectors of the upper or lower surface } \\
& i \quad \text { the crack opening increment index , } \\
& \text { CTODincr is the commanded increment of CTOD in one process step, } \\
& \text { slope is a calculated process parameter based on matching initial and final crack } \\
& \text { profiles as described above, } \\
& Y \quad \text { are the data position vectors along the crack extension direction, and } \\
& R_{i} \quad \text { is the calculated rotational center position ahead of the crack tip. }
\end{aligned}
$$

The sign of the incremental opening and rotation slope is reversed when manipulating the opposite surface data set, such that the "upper" surface moves up and the "lower" surface moves down, and the applied rotational correction is in opposite directions. $\mathrm{R}$ is based on the center of rigid rotation predicted by equation A1.13 or A2.16 in ASTM E 1820 [10], and the crack tip position, $\mathrm{a}_{\mathrm{i}}$, after the previous opening increment. The values in $\mathrm{Y}$ are adjusted to account for change in position due to the rotation. Although this correction is quite small in most cases, it is included for accurate profile shape representation in the case of very large openings and rotations that may be encountered with very tough, very ductile materials.

Calculated crack profile shapes generated using this process equation can be displayed so that the evolution of the crack opening and extension can be visualized. Various methods, based on the different definitions of CTOA, are applied to the incremental profiles to determine the CTOA at that particular state. If CTOA is determined according to one of the standard definitions, and local slant cracking is not to be considered, the height difference function, $D$ (Eqn 2) can be calculated from upper and lower profile data, simplifying the analysis. The crack extension at the corresponding increment is determined based on the position of intersection of the upper and lower surface profiles (where the sign of $D$ becomes negative). While this approach to crack tip position determination does not explicitly account for process zone tip shape, the potential errors are very small compared to the conventional average crack length assessment using compliance or electric potential methods. 
This correction of crack face rotations is an important consideration when deriving CTOA from a fixed location on the specimen or fracture surface, for example at the original crack tip as with the $\delta_{5}$ definition. It is of less importance when using a position a fixed distance behind the instantaneous tip location, such as $1.0 \mathrm{~mm}$, but still may be a source of significant error. When making this opening displacement measurement at increasing distances behind the crack tip, there is a potential for incurring larger errors. The direct result of the specimen rotation during crack growth will be an artificial increase in measured CTOA with increasing crack extension. Assuming a perfectly constant CTOA, the rotation will cause the "planar" surfaces become somewhat convex, resulting in measured openings at some distance behind the crack tip to be larger than the "true" opening.

A different part of the fracture process - plastic material flow lines intersecting the crack flanks behind the crack tip - has the potential to create the opposite effect. Based on non-hardening slip line plasticity analysis, this effect would be most apparent when the crack flank angles become increasingly larger. This is demonstrated in Figures 37, 38, and 39 on page 146 in Fracture: An Advanced Treatise [11]. Although not typical of a normal fracture toughness test specimen, notches or angled grooves on the surface opposite to the crack may exaggerate the effect (see Slip Line Analysis later). This type of material flow will result in flat crack flank surfaces becoming concave, and the resulting measured CTOA will be lower than the "true" value representing deformation processes occurring in the tip process zone. Alternatively, the tip process zone could be, and maybe should be, enlarged to encompass the entire region of plastic flow behind the crack tip.

Both of these effects cause worsening errors with increasing crack extension, and with increasingly larger measurement distances behind the instantaneous crack tip location. The one positive aspect is that these measurement errors have opposite effect on the measured CTOA, tending to cancel each other's effects. The magnitude of these effects has not been quantified. Special specimen geometries that are predisposed to the plastic flow of interest will need to be tested and analyzed so the effects can be measured.

\section{Crack Face Rotations - The Good News}

Microtopography allows the CTOA parameter to be assessed using various definitions. For example, varying "gage" distances behind the crack tip can be used, and the resulting effect on measured CTOA for a given amount of crack extension can be determined. By confining measurements to the near-tip region - but beyond the estimated process zone boundary - errors due to specimen anti-symmetric rotation remain somewhat constant, even if surface angle corrections to account for the rotations are not applied.

Another factor is related to the general toughness and ductility of materials. Materials with lower toughness (lower tearing modulus) tend to have a correspondingly smaller CTOA response characteristic. The general ductility of these materials is also usually lower and yield strengths are frequently higher. The combined result of lower CTOA of the growing crack and reduced plastic deformation of the ligament ahead of the crack tip is reduced rigid rotation of the two crack flanks. Therefore, for a given crack extension the corresponding specimen global rotation is also smaller. The converse is true for tougher materials with higher CTOA - larger rotations for a given amount of crack extension. Therefore, the net error attributed to rigid anti-symmetric crack flank rotation is essentially a fixed percentage of the measured CTOA. Stated differently, the effect of the crack flank rotation is an invariant process error for a particular type of test specimen geometry. 


\section{Assessment of Accuracy - Experiments and Slip Line Analysis}

The present implementation of microtopography analysis of the CTOA response considers crack opening profiles at individual, selected cross-sections of the fracture surfaces. A planar "slice" normal to the average crack plane and parallel to the nominal crack growth direction is selected from the three-dimensional data set. The slice data are then manipulated using Equation 2 or 4 to re-create the fracture process at that slice plane. The processing algorithm allows the crack mouth opening displacement (CMOD) corresponding to a given increment or state of local crack opening to be inferred by extrapolating the opening displacement to the specimen surface location. The CMOD value is a direct result of computed center of rotation and crack tip location for the particular state of the profile "slice" being examined. Consider various slices taken at different through thickness positions, and the fact that the crack border is curved in a typical bend-type fracture test specimen. For any specimen fracture process state considered, the rigid motion of the specimen away from the crack tip dictates that the CMOD be a constant at that state. Therefore, for any profile section considered, the calculated CMOD can serve to connect the analysis of one profile position to any other. Further, the rigid far-field behavior of the test specimen mandates a constant position (relative to the specimen surface, for example) for center of rotation at a given fracture process state. This condition is required regardless of the profile location through the specimen thickness. These physical constraints were used to assess the accuracy of the microtopographic process model.

The terminal state of the ductile fracture process is marked by fatigue cracking or cleavage fracture. This state is readily identified in any profile section considered. The plastic component of CMOD at this state is also known, measured by a CMOD clip gage following specimen unloading. While performing independent process modeling of various profile sections in a particular specimen, a steel weldment $\mathrm{SE}(\mathrm{B})$-type specimen with $\mathrm{B}=\mathrm{W}=25 \mathrm{~mm}$, the end state CMOD value determined by the microtopography process matched the experimentally measured values (by clip-on gage during the test) within 5 percent. That provides confidence in the accuracy of the process model equations. This allows the inferred CMOD values from analyses of the various profile positions to be used to match process states between them. At any particular process state, the calculated center of rotation for any given profile section can then be compared to the others. If everything in the re-creation process is functioning properly, the center of rotation positions should match. Early results of our investigation show variations the order of 2 percent of the specimen width, $\mathrm{W}$, to be typical. These independent comparisons of physical phenomena provide assurance that the fracture process model is satisfactory. Accurate representations of actual crack opening profile shapes during the fracture process are generated by the microtopography analysis, and the local parameters extracted from the individual profile sections can be relied upon.

\section{Slip Line Analysis - Common Center of Rotation Assumption}

McClintock performed an initial assessment of the center-of-rotation correction applied in the microtopography analysis of CTOA. Slip line fracture mechanics (SLFM) were applied to analyze the single edge cracked bend specimen described in the above section "Assessment of Accuracy - Experiments and Slip Line Analysis." SLFM uses non-hardening plasticity assumptions, but still predicts maximum errors the order of $10 \%$ in position of effective center of rotation for the SE(B) specimen with mid-depth cracks. This provides additional assurance that the re-creation model equations being applied in the microtopography methodology are reasonable and provide results with sufficient accuracy. Material hardening effects and other specimen geometries with very different slip line fields may, however, result in different results 
that reduce the reliability of the microtopography CTOA predictions. Work in this area of verification of the microtopography method is continuing.

\section{Microtopography Methodology Summary}

Microtopography allows a full three-dimensional assessment of the ductile fracture process, including the crack tip opening angle. The method allows many states to be interrogated, from tip blunting before actual ductile tearing crack growth, through extensive ductile crack growth - all using only a single test specimen. The process model has been developed to account for antisymmetric rigid specimen rotation during crack growth if it occurs. The re-creation process has proven to be consistent when center of rotation positions, calculated from individual profile slices from different locations within a single specimen, are compared to one another at equivalent states (CMOD). An analytical method has been developed to extract the local crack tip opening angle, using any of several definitions of CTOA. This method was applied to analyze CTOA in various test specimens. Accuracy of results of the analyses compared favorably to other conventional methods of CTOA determination $[2,3]$. The microtopography method offers two unique advantages in ductile fracture analysis: interrogation of fracture process variables, e.g. CTOA and $\Delta \mathrm{a}$, within the specimen interior; and, process analysis entirely after the actual ductile fracture process is completed.

\section{Acknowledgement}

This work was supported by the U.S. Department of Energy, Office of Science, Office of Basic Energy Sciences, Engineering Research, under DOE Idaho Operations Office Contract DE-AC07-99ID13727.

\section{References}

1. Dawicke DS, Newman JC Jr., Bigelow CA. Three Dimensional CTOA and constraint effects during stable tearing in a thin-sheet material. In: Reuter WG, Underwood JH, Newman JC Jr., editors. Fracture Mechanics: $26^{\text {th }}$ Volume, ASTM STP 1256, American Society for Testing and Materials, Philadelphia, 1995. pp. 223-242.

2. Sutton MA, Dawicke DS, Newman JC Jr. Orientation effects on the measurement and analysis of critical CTOA in an aluminum alloy sheet. In: Reuter WG, Underwood JH, Newman JC Jr., editors. Fracture Mechanics: $26^{\text {th }}$ Volume, ASTM STP 1256, American Society for Testing and Materials, Philadelphia, 1995. pp. 243-255.

3. Lloyd WR, Piascik RS. Three-dimensional crack growth assessment by microtopographic examination. In: Reuter WG, Underwood JH, Newman JC Jr., editors. Fracture Mechanics: $26^{\text {th }}$ Volume, ASTM STP 1256, American Society for Testing and Materials, Philadelphia, 1995. pp. 303-318.

4. Reuter WG, Lloyd WR. Measurements of CTOD and CTOA around surface-crack perimeters and relationships between elastic and elastic-plastic CTOD values. ASTM Special Technical Publication, STP 1060, April 1990, American Society for Testing and Materials, Philadelphia, PA, p. 152.

5. Guan Z-X, Zhang Z-L, and Yang X-H. Initial analysis of the local field in the vicinity of a stably growing crack-tip and the correspondent fracture parameters. Engineering Fracture Mechanics, v 34, n 2, 1989, pp. 507-523. 
6. James MA and Newman JC, Jr. Characterization of crack length measurement methods for flat fracture with tunneling. In: Fatigue and Fracture: $33^{\text {rd }}$ vol., ASTM STP 1417, American Society for Testing and Materials, West Conshohocken, PA, 2002.

7. Zerbst U, Heerens J, and Schwalbe K-H. Fracture mechanics analysis based on a local simulation principle. Fatigue and Fracture of Engineering Materials \& Structures, v18, n3, March 1995, University of Sheffield, Sheffield, UK, p 371-376.

8. Zerbst U, and Schwalbe K-H. ... , Engrg Frac Mech, v??, n??, pp. ??-??, 2002.

9. Lloyd WR. Microtopography for Ductile Fracture Process Characterization - Part 1: Theory and Methodology, Engrg Frac Mech, v??, n??, pp. ??-??, 2002.

10. Standard Test Method for Measurement of Fracture Toughness, Annexes A1 - A3, ASTM E 1820-99, Annual Book of Standards, v.3.01, American Society for Testing and Materials, W. Conshohocken, PA, 1999, pp. 983-990.

11. McClintock, FA. Plasticity Aspects of Fracture, in Fracture: An Advanced Treatise, vol. 3, H. Liebowitz, ed., Academic Press, New York, 1971, p. 146. 
Figures 


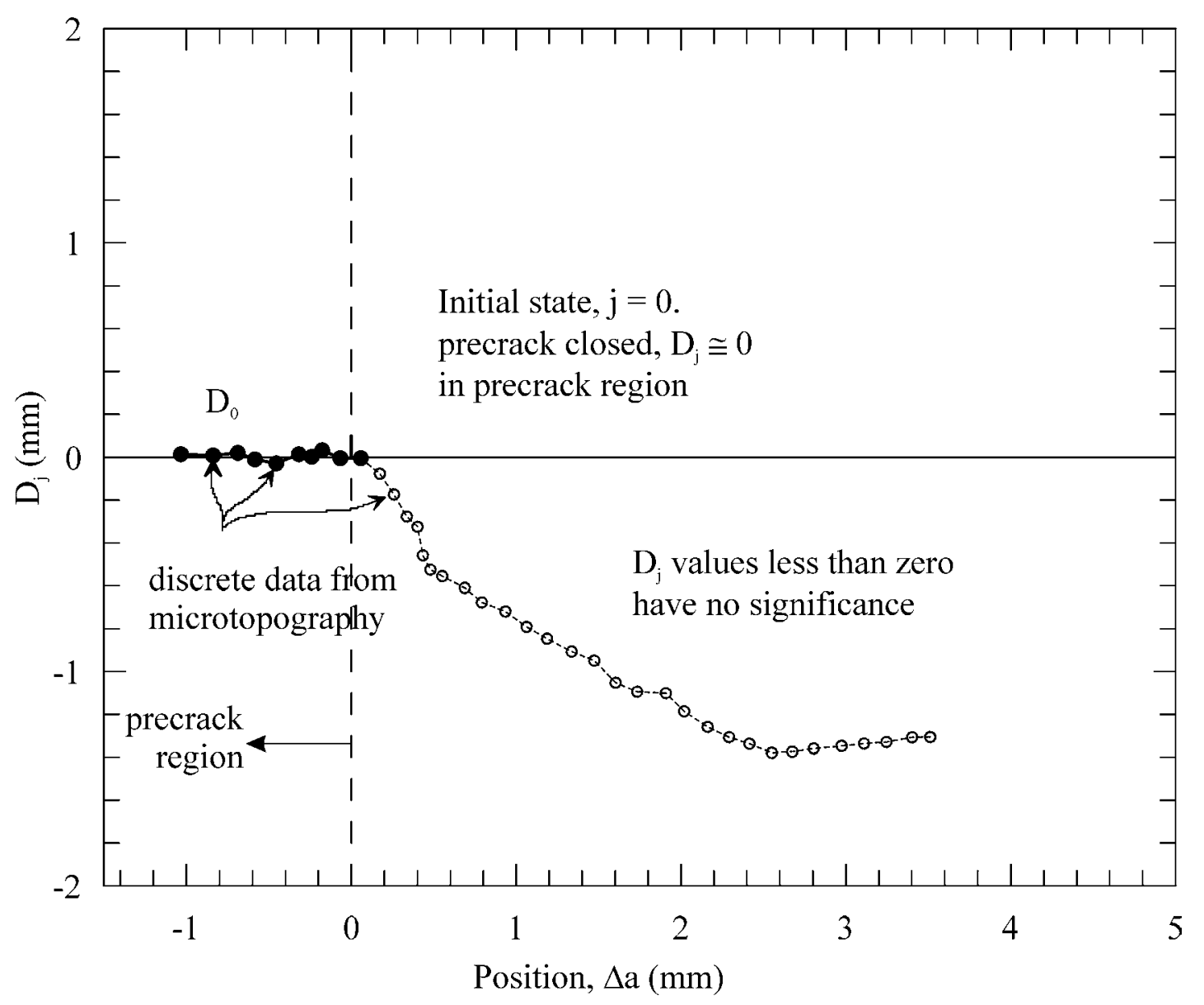

Figure 1. Representation of the crack opening separation, $\mathrm{D}=\mathrm{D}_{0}$, at initial state (no crack opening) with separation in precrack region set to 0 . Raw data (U and $L$ heights) taken in plane parallel to crack opening and parallel to crack growth near specimen mid-thickness. 


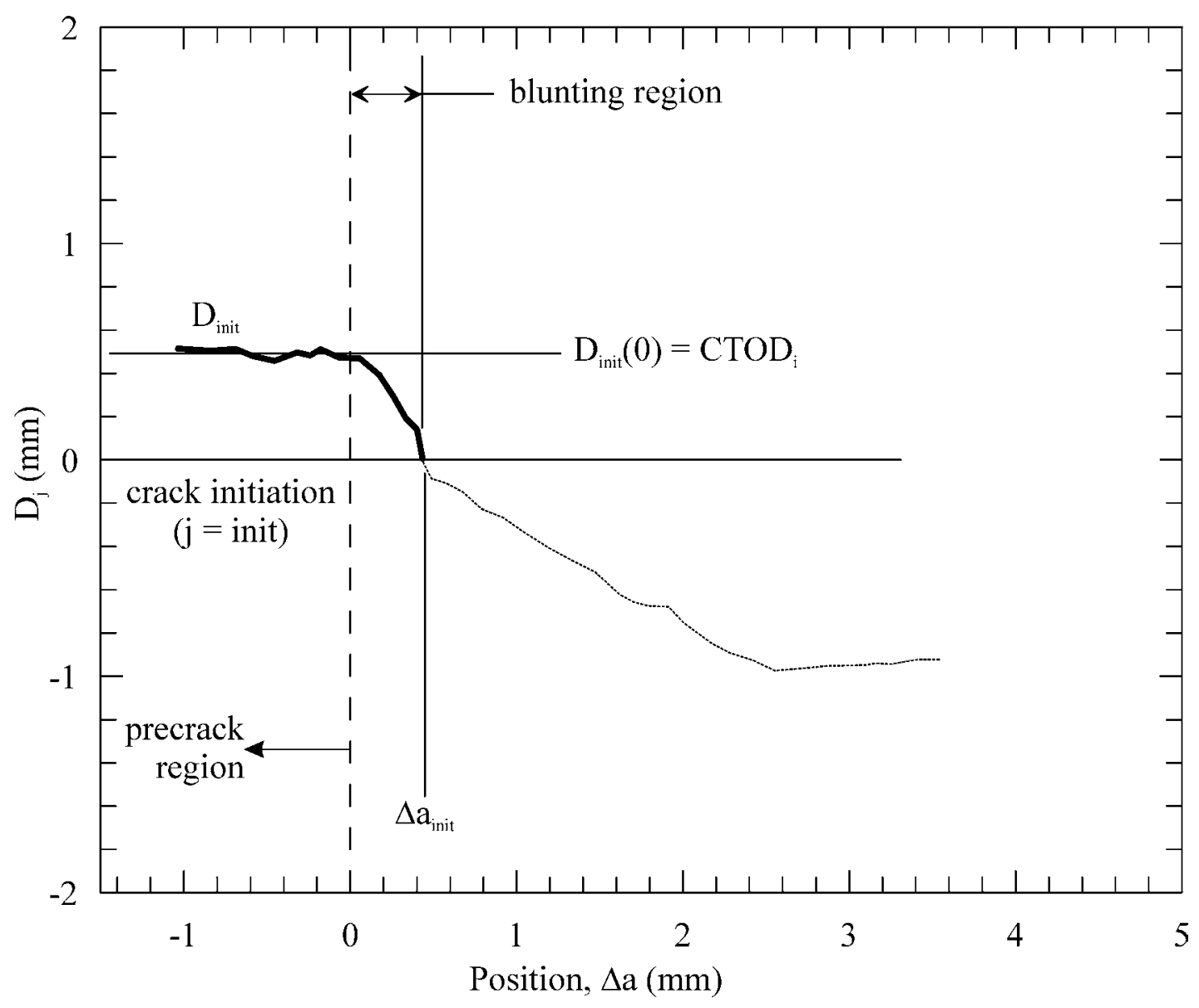

Figure 2. Representation of the crack opening separation, $D=D_{\text {init }}$, at crack initiation state. $\mathrm{CTOD}_{\mathrm{i}}=\mathrm{D}(\Delta \mathrm{a}=0)$. Data is from an $\mathrm{SE}(\mathrm{B})$-type bend specimen. The $\mathrm{D}$ function undergoes opening translation (vertical on graph), as well as some rigid rotation as determined by instantaneous crack tip position (crack length) and applied opening increment. This rotation continues in Figures 3 - 5 . 


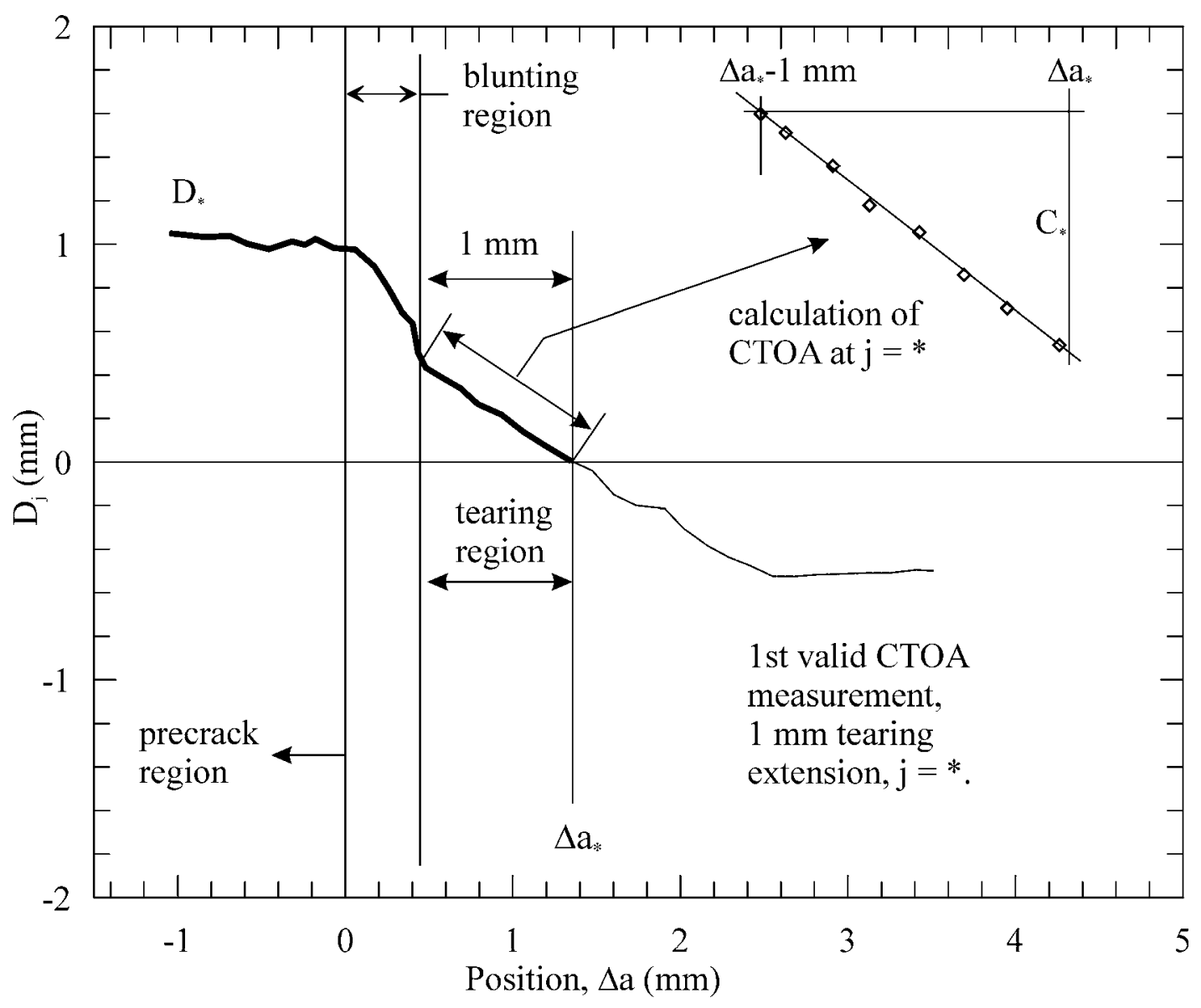

Figure 3. Representation of the crack opening separation, $\mathrm{D}=\mathrm{D}_{*}$, at $1 \mathrm{~mm}$ of ductile tearing extension. CTOA determined by the " $1 \mathrm{~mm}$ " definition is shown. Note, a single point measurement at $\mathrm{D}=\mathrm{D}(\Delta \mathrm{a}-1 \mathrm{~mm})$ could be used to determine $\mathrm{C}$, but using a linear curve fit of data over a range of $\Delta$ a reduces variability in the calculated slope values, $C$, due to original height measurement error and or slight positional mis-registration. 


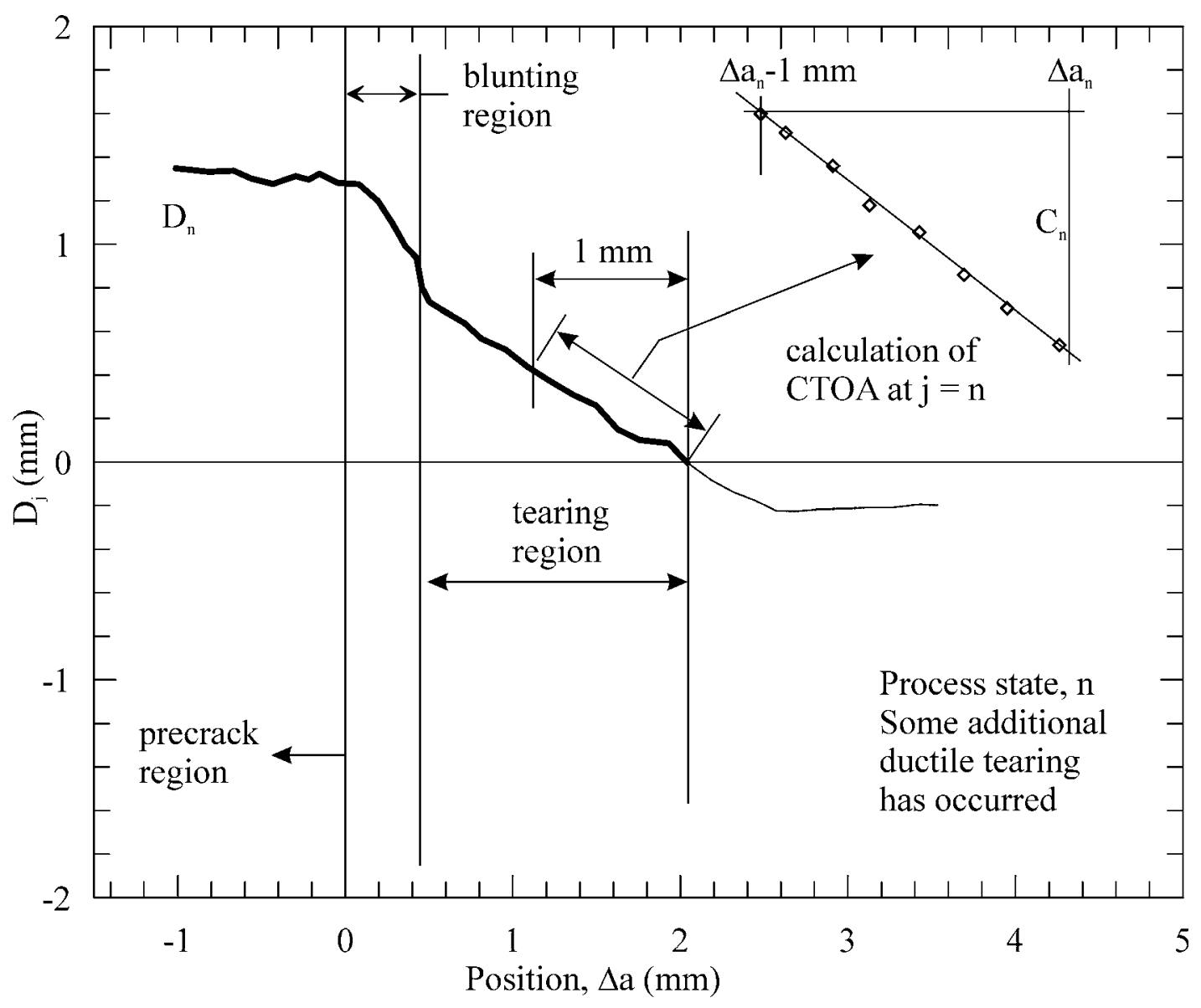

Figure 4. Representation of the crack opening separation, $D=D_{n}$, at $2 \mathrm{~mm}$ of ductile tearing extension. CTOA determined by the " $1 \mathrm{~mm}$ " definition is also shown. 


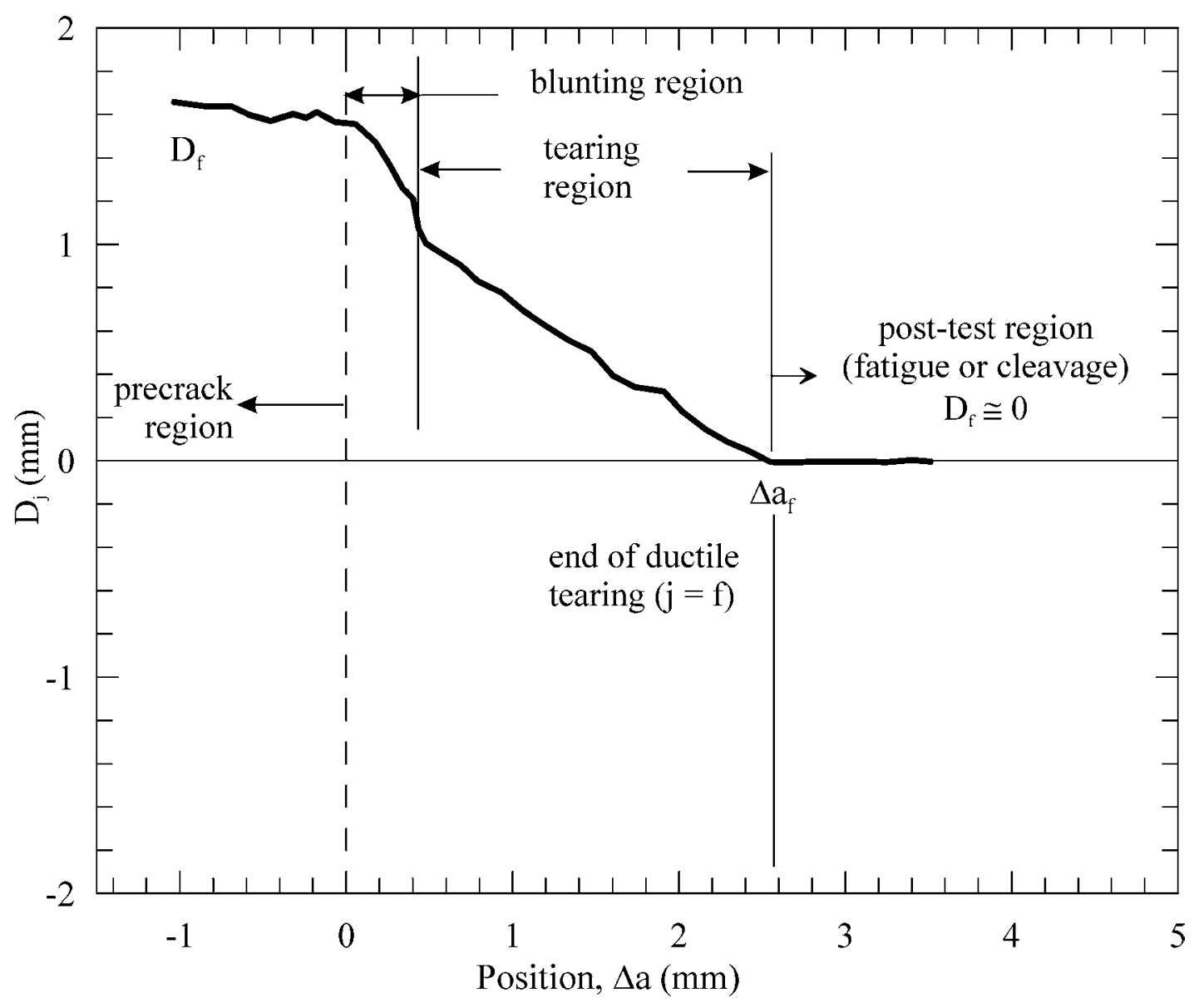

Figure 5. Representation of the crack opening separation, $\mathrm{D}=\mathrm{D}_{\mathrm{f}}$, at conclusion of ductile tearing extension. Height data collected in the post-test cleavage fracture region shows a nominal separation of 0 , due to elastic nature of the cleavage fracture. This final state, along with the initial state shown in Figure 1, is used to calibrate the rigid rotation correction applied in the analysis. 


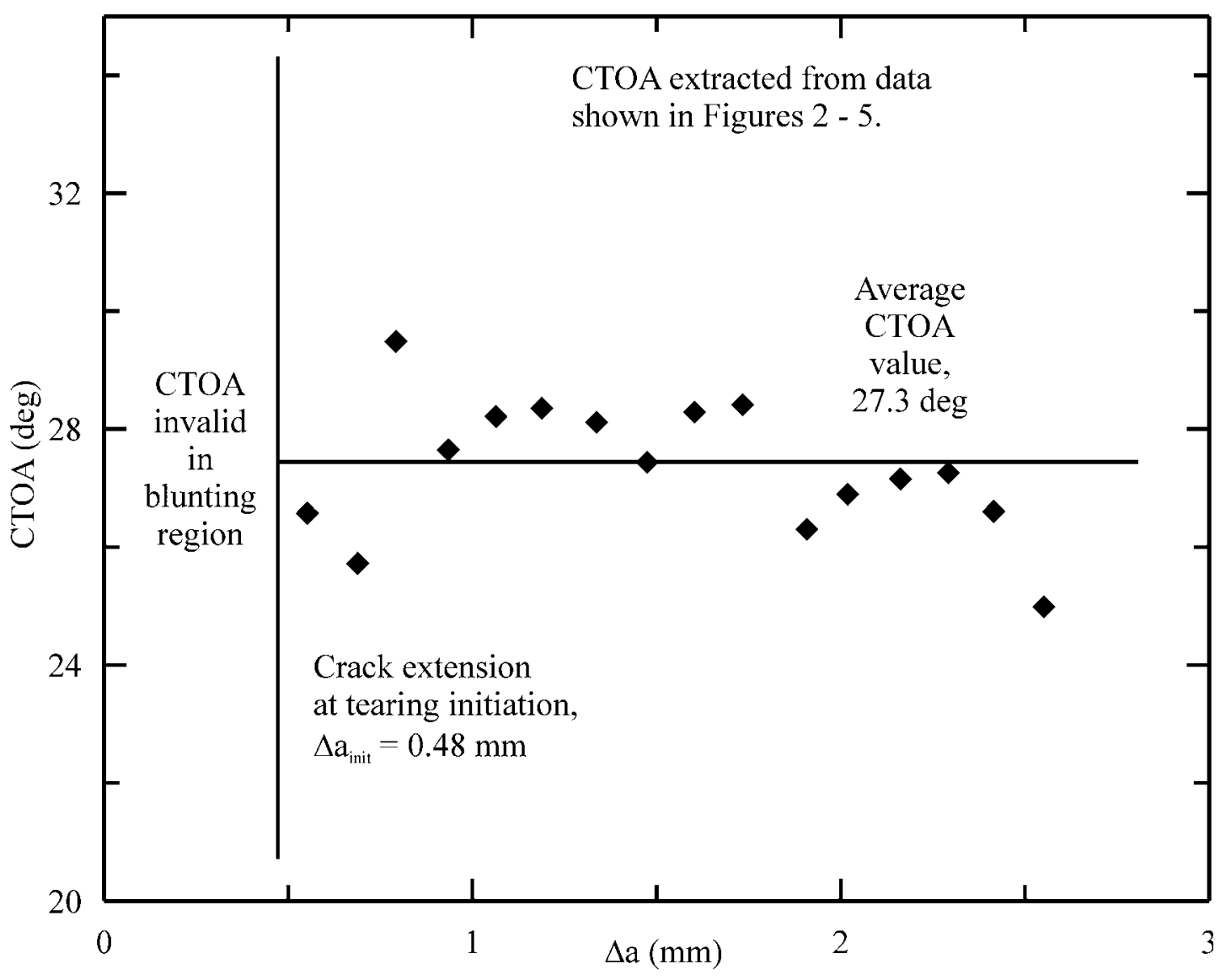

Figure 6. Final analysis of the variation of CTOA with ductile crack extension. The region of crack extension between $\Delta \mathrm{a}=0$ and $0.5 \mathrm{~mm}$ is associated with crack tip blunting (stretch zone) where CTOA is not a meaningful parameter to characterize the crack tip material response. 